\title{
Synthesis of 2-thioxoimidazolin-4-one and thiazolo[3,2-a]- benzimidazole derivatives from substituted maleimides
}

\author{
Yamina Bentarzi, ${ }^{\text {a,b }}$ Bellara Nedjar-Kolli, ${ }^{\mathrm{a} *}$ Aurélie Plas, ${ }^{\mathrm{c}}$ Pierre Chalard, ${ }^{\mathrm{a}}$ and Yves Troin ${ }^{\mathrm{c}}$ \\ ${ }^{a}$ Laboratoire de Chimie Organique Appliquée, Université des Sciences et Techniques Houari \\ Boumediene, BP 3, El-Alia, Bab-Ezzouar, 16111, Alger, Algérie \\ ${ }^{b}$ Centre de Recherche Scientifique Technique en Analyse Physico-chimique (CRAPC) BP248 \\ Alger RP, 16004, Algérie \\ ${ }^{c}$ Clermont Université, ENSCCF, EA 987, LCHG, BP 10448, F-63000 Clermont-Ferrand, France \\ E-mail: yaslydia2005@yahoo.fr
}

\begin{abstract}
We describe here a hetero Michael-type reaction, involving maleimides and 2cyanoacetichydrazide or 2-mercaptobenzimidazole, followed by an opening cyclization reaction with isothiocyanates. This reaction allows the preparation of new 2-thioxoimidazolidin-4-one and thiazolo[3,2-a]benzimidazole derivatives depending on the nucleophile used.
\end{abstract}

Keywords: Thioxoimidazolin-4-one, hetero Michael reaction, thiazolobenzimidazoles, maleimide

\section{Introduction}

2-thioxoimidazolin-4-one (thiohydantoins) framework is often encountered in compounds dealing with biological activity. As examples, thiohydantoin $\mathbf{1}$ is an efficient inhibitor of muscle and liver glycogen phosphorylases ${ }^{1}$ and has also showed herbicidal activity by inhibition of purine biosynthesis. ${ }^{2}$ Substituted 4-methylene-2-thiohydantoins 2 have displayed Cycline Dependant Kinases (CDK) inhibition in a micromolar range $\mathrm{e}^{3 \mathrm{a}}$ or antileishmanial activity ${ }^{3 \mathrm{~b}}$ whereas compounds 3 are Fatty Acid Amide Hydrolase (FAAH) inhibitors ${ }^{4}$ and compounds 4 are claimed to be active for treatment of hormone refractory prostate cancer. ${ }^{5}$

So, it is not surprising if a lot of patents have described the use of this attractive skeleton for a range of activity which cover from compounds for biological use ${ }^{6}$ till fire retardants materials. ${ }^{7}$ Besides conventional methods used for their synthesis, solid phase, ${ }^{8}$ use of ionic liquids ${ }^{9}$ and microwaves-assisted approaches have been published. ${ }^{10}$ On the other hand, the benzimidazole structure is also a scaffold often endowed with different types of biological activities including antitumor, $^{11}$ antimicrobial $^{12}$ or antiviral $^{13}$ properties. Thiazole nucleus fused with a 
benzimidazole skeleton, namely thiazolobenzimidazoles, have been reported to exhibit biological properties too, such as anti-HIV ${ }^{14}$ or anti-microbial ${ }^{15}$ agents. We wish to report here the synthesis of new 2-thioxoimidazolin-4-one and thiazolobenzimidazoles derivatives by hetero Michael reactions on $N$-substituted maleimides.

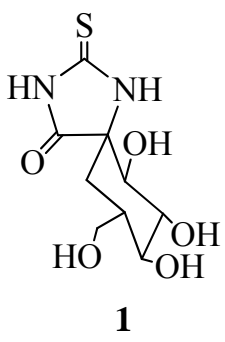

1

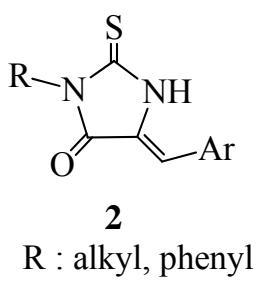

$\mathrm{R}$ : alkyl, phenyl<smiles>[X]c1ccc(C2(c3ccc([X])cc3)NC(=S)N([R])C2=O)cc1</smiles>

3

$\mathrm{R}$ : alkyl, phenyl

$\mathrm{X}$ : halogen<smiles>[R2]C1([R])C(=O)N([Al])C(=S)N1[Al]</smiles>

4 $\mathrm{R}_{1}, \mathrm{R}_{2}$ : alkyl, phenyl

Figure 1. Thiohydantoins with described biological activities.

\section{Results and Discussion}

\section{2-Thioxoimidazolin-4-one derivatives}

Previous investigations from our laboratory ${ }^{16}$ have shown that 2-thioxoimidazolin-4-one derivatives 7 could be easily obtained by Michael addition of 3-ethylhydrazinoacetate on $\mathrm{N}$ substituted maleimide 5, leading to 3,4-dihydro maleimides 6 which reacted smoothly with substituted isothiocyanates to give compounds 7 (Scheme1).
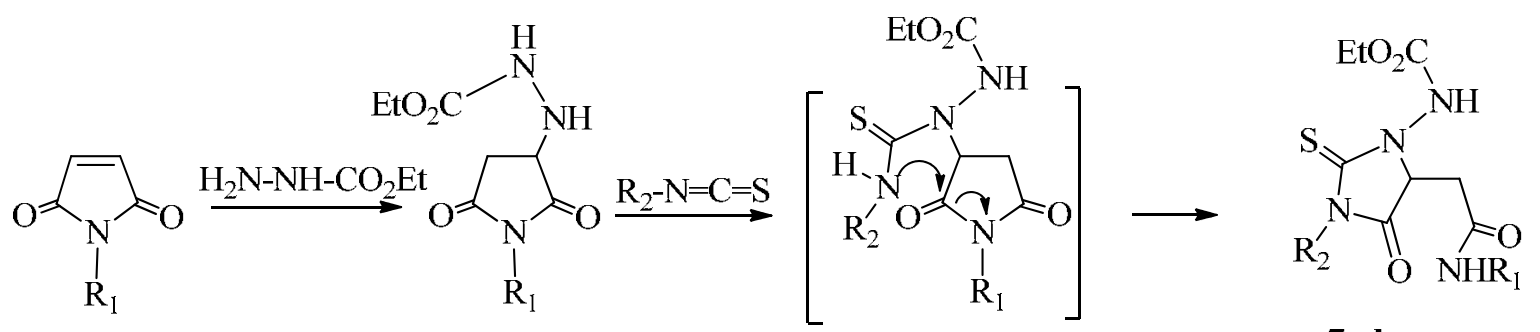

5a $\quad \mathrm{R}_{1}=\mathrm{H}$

5b $\quad \mathrm{R}_{1}=\mathrm{CH}_{3}$

6a-d

5e $\quad \mathrm{R}_{1}=\mathrm{C}_{2} \mathrm{H}_{5}$

$\mathrm{R}_{\mathrm{l}}=\mathrm{H}, \mathrm{CH}_{3}, \mathrm{C}_{2} \mathrm{H}_{5}, \mathrm{C}_{6} \mathrm{H}_{5}$

5d $\quad \mathrm{R}_{\mathrm{I}}=\mathrm{C}_{6} \mathrm{H}_{5}$

Scheme 1. Synthesis of compounds 7a-h.

We wish to report here an extension of this methodology using 2-cyanoacetic hydrazide as nucleophile. This compound, by its carbonyl and cyano functions, is enabling to react with 
bidendate reagents. Moreover, the active hydrogen of this molecule can also take part in a wide range of condensation and substitutions reactions. ${ }^{17}$

Thus, condensation of maleimide 5a-d with 2-cyanoacetic hydrazide in refluxing ethanol led to compounds 8a-d in fair to good yields. Spectral data $\left({ }^{1} \mathrm{H}\right.$ and ${ }^{13} \mathrm{C}$ NMR) were in complete accordance with the proposed structures and showed that these compounds existed under a tautomeric equilibrium between the keto (I) and enol (II) form in an average ratio of 3/1. For example concerning succinimide 8d, protons of $\mathrm{CH}_{2}-\mathrm{CH}$ fragment showed characteristic patterns of an ABX system (Scheme 2).

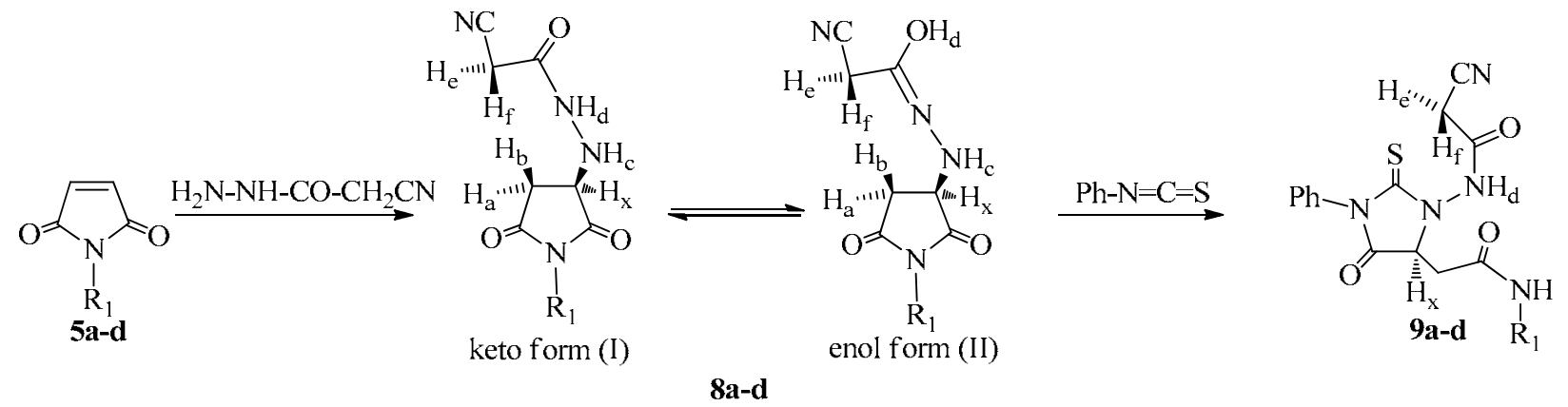

Scheme 2. Preparation of 2-thioxoimidazolidines 9a-d.

The chemical shift observed for the doublet of doublet corresponding to proton $\mathrm{H}_{\mathrm{a}}$ was $\delta=$ $2.69 \mathrm{ppm}(J=4$ and $18 \mathrm{~Hz})$ in the form (I) while it was $\delta=2.85 \mathrm{ppm}(J=5$ and $18 \mathrm{~Hz})$ in the form (II). For proton $\mathrm{H}_{\mathrm{b}}$, the observed chemical shift was $\delta=3.05 \mathrm{ppm}(\mathrm{J}=8$ and $18 \mathrm{~Hz})$ in form (I) and $2.95 \mathrm{ppm}(J=8$ and $18 \mathrm{~Hz})$ in form (II). Same observations could also be made on protons $\mathrm{H}_{\mathrm{e}}$ and $\mathrm{H}_{\mathrm{f}}$. In form $(\mathrm{I}) \mathrm{H}_{\mathrm{e}}$ and $\mathrm{H}_{\mathrm{f}}$ give two doublets respectively at $3.62\left(\mathrm{H}_{\mathrm{e}}\right)$ and $3.67\left(\mathrm{H}_{\mathrm{f}}\right)$ ppm $(J=18 \mathrm{~Hz})$, while in form (II) the signals corresponding to these protons was shifted to $3.90\left(\mathrm{H}_{\mathrm{e}}\right)$ and $4.08\left(\mathrm{H}_{\mathrm{f}}\right)$ ppm $(J=19 \mathrm{~Hz})$. As described previously, condensation of succinimides 8a-d with phenylisothiocyanate furnished the corresponding 2-thioxoimidazolidines 9a-d resulting of the opening-recyclization reaction. For these compounds $\mathbf{9}$, it should be noted that no tautomeric form was observed, contrary to compounds 8. Here too, the structures of these new compounds were confirmed by careful examination of spectral data, showing mainly in the IR spectra a band around $v=1500 \mathrm{~cm}^{-1}$ and in the ${ }^{13} \mathrm{C}$ NMR spectra a signal at $184 \mathrm{ppm}$ attributed to the thiocarbonyl group.

Next, we envisaged to test the reactivity of the 2-cyanoacetic appendage. So, Knoevenagel condensation of compound 8d with benzaldehyde in refluxing ethanol containing a catalytic amount of piperidine ${ }^{18}$ resulted in the formation of benzylidene $\mathbf{1 0}$ which reacted smoothly with phenylisothiocyanate to furnish the 2-thioxoimidazolidine derivative $\mathbf{1 1}$ in good yield (Scheme 3 ), the structure of which was in accordance with all spectral data. 


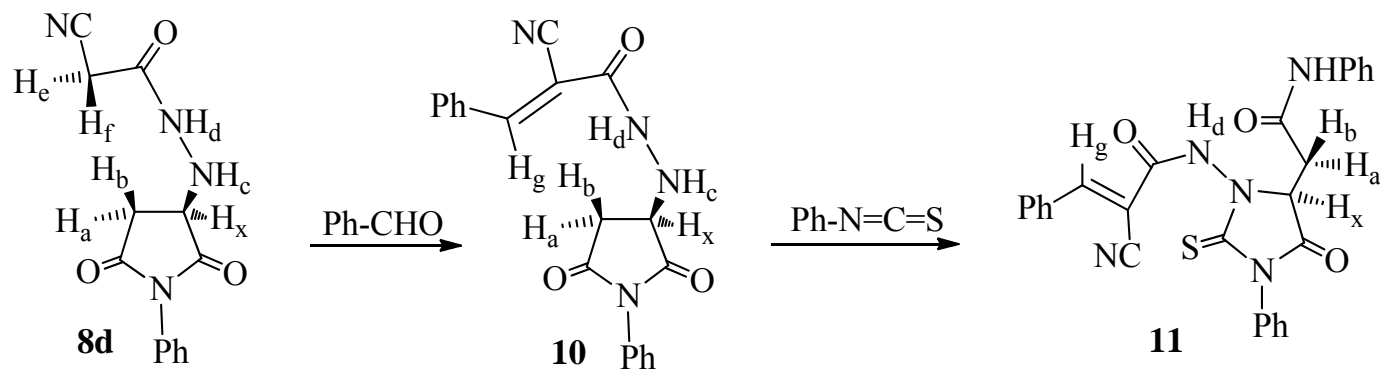

Scheme 3. Synthesis of compound 11.

\section{Thiazolo[3,2-a]benzimidazole derivatives}

Finally we envisaged to test the reactivity of other nucleophiles on hetero Michael addition to maleimide and for this purpose we tried the addition of 2-mercaptobenzimidazole 12. This latter was chosen as this framework is endowed with a lot of biological activities. ${ }^{11,13}$ Several methods have been developed for the synthesis of thiazolo[3,2-a]framework using the nucleophilicity of the thiol function of 2-mercaptobenzimidazole. ${ }^{19}$ A recent work has also described the synthesis of target molecule by opening thiiranes with 2-chlorobenzimidazole. ${ }^{20}$ Thus maleimides 1a,b,d reacted with 12 in refluxing acetic acid in presence of concentrated sulphuric acid to afford in each case the corresponding thiazolobenzimidazole 13a,b,d in average yields (Scheme 4).<smiles>[R]N1C(=O)C=CC1=O</smiles>

$5 a, b, d$<smiles>Sc1nc2ccccc2[nH]1</smiles>

12
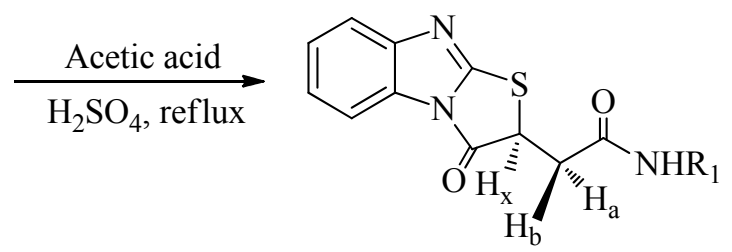

Scheme 4. Synthesis of thiazolobenzimidazole 13a,b,d.

Formation of compounds $\mathbf{1 3}$ resulted from initial attack of the mercapto group on the double bond of maleimide followed, as described previously, by ring opening of the maleimide by the amino group of the benzimidazole ring. Spectral data obtained from compounds $\mathbf{1 3}$ were in complete accordance with the expected thiazolobenzimidazole structures: in ${ }^{1} \mathrm{H}$ NMR spectrum of each derivative, together with the expected signals, one can noticed the presence of three new signals at around 2.95, 3.30-4.95 and $11.30 \mathrm{ppm}$, due respectively to $\mathrm{CH}, \mathrm{CH}_{2}$ and $\mathrm{NH}_{2}$ protons. Moreover in ${ }^{13} \mathrm{C}$ NMR spectrum, signals at 38.6, 62.4 and $177.9 \mathrm{ppm}$ were assigned to $\mathrm{CH}, \mathrm{CH}_{2}$ and $\mathrm{C}=\mathrm{O}$ carbons beside all others carbons at the expected regions.

In conclusion, we have described new 2-thioxoimidazolidine and new thiazolo[3,2-a]benzimidazole derivatives. The use of 2-cyanoacetic hydrazide as nucleophile in the heteroMichael reaction on maleimide, followed by the reaction with isothiocyanates, has permitted an entry to polyfunctionalized heterocyclic structures due to its intrinsic reactivity. The extension of 
this methodology, together with the use of the variety of reactions which could be realized ${ }^{21}$ starting from this brick, is actually under progress.

\section{Experimental Section}

General. Unless otherwise specified, reagents were obtained from commercial suppliers. Solvents were dried and freshly distilled following the usual procedures. Product organic solutions were dried over sodium sulfate prior to evaporation of the solvents under reduced pressure on a rotatory evaporator. Thin layer chromatography was performed on TLC precoated aluminium backed silica plates and spots were visualized using UV light (254 nm) before using ethanolic phosphomolybdic acid solution (heating). Column chromatography was carried out on silica gel (70-230 mesh).Melting points were measured in open capillary tubes on a BÜCHI B540 apparatus. ${ }^{1} \mathrm{H}$ and ${ }^{13} \mathrm{C}$ NMR spectra were measured at 400.13 and $100.61 \mathrm{MHz}$ respectively using TMS as internal reference. Chemicals shifts are reported in ppm relative to TMS. Signals are quoted s (singlet), d (doublet), t (triplet), q (quartet), m (multiplet), br (broad) and coupling constant $(\mathrm{J})$ values are given in Hz. Infrared spectra were recorded on a Shimadzu FTIR 8400s spectrophotometer. High Resolution Electro Spray Impact Mass Spectra (HR-ESI-MS) were obtained from the Centre Régional de Mesures Physiques de l'Université Blaise Pascal (Clermont II), France. Elemental analyses (C, H, O, N) were performed at the Centre de Microanalyses du CNRS, Lyon, France.

\section{General procedure for preparation of compounds (8)}

A mixture of cyanoacetic acid hydrazide (1 equivalent) and the corresponding maleimide 5a-d ( 1 equivalent) in ethanol was refluxed for 12 hours. After cooling the precipitate was filtered and the resulting solid was crystallized from methanol.

2-Cyano- $N^{\prime}$-(2,5-dioxopyrrolidin-3-yl)acetohydrazide (8a). Yield 52\%; white solid; mp 102$103{ }^{\circ} \mathrm{C}$, IR (KBr, $\left.v \mathrm{~cm}^{-1}\right)$ ) $3348(\mathrm{~m}), 3282(\mathrm{w}), 3194(\mathrm{~m}), 3136(\mathrm{w}), 2260(\mathrm{~m}), 1703$ (s), 1685 (s), $1622(\mathrm{~m}) ;{ }^{1} \mathrm{H}$ NMR (400 MHz, DMSO-d $) \delta 11.25$ (1H, br s, NH (I, II)), $9.68\left(1 \mathrm{H}, \mathrm{d},{ }^{3} J=5.5\right.$ $\left.\mathrm{Hz}, \mathrm{H}_{\mathrm{d}}(\mathrm{I})\right), 9.07\left(1 \mathrm{H}, \mathrm{s}, \mathrm{H}_{\mathrm{d}}(\mathrm{II})\right), 5.67\left(1 \mathrm{H}, \mathrm{d},{ }^{3} J=3.5 \mathrm{~Hz}, \mathrm{H}_{\mathrm{c}}(\mathrm{II})\right), 5,64\left(1 \mathrm{H}, \mathrm{t},{ }^{3} J=4.5 \mathrm{~Hz}, \mathrm{H}_{\mathrm{c}}(\mathrm{I})\right)$, 4.05-3.95 (3H, m, $\mathrm{H}_{\mathrm{x}}(\mathrm{I}, \mathrm{II})$ and $\left.\mathrm{H}_{\mathrm{f}}(\mathrm{II})\right), 3.86\left(1 \mathrm{H}, \mathrm{d},{ }^{2} J=19.0 \mathrm{~Hz}, \mathrm{H}_{\mathrm{e}}(\mathrm{II})\right), 3.63\left(1 \mathrm{H}, \mathrm{d},{ }^{2} J=18.0\right.$ $\left.\mathrm{Hz}, \mathrm{H}_{\mathrm{f}}(\mathrm{I})\right), 3.58\left(1 \mathrm{H}, \mathrm{d},{ }^{2} J=18.0 \mathrm{~Hz}, \mathrm{H}_{\mathrm{e}}(\mathrm{I})\right), 2.80\left(1 \mathrm{H}, \mathrm{dd},{ }^{2} J=18.0,{ }^{3} J=8.0 \mathrm{~Hz}, \mathrm{H}_{\mathrm{b}}(\mathrm{I})\right), 2.77$ $\left(1 \mathrm{H}, \mathrm{dd},{ }^{2} J=18.0,{ }^{3} J=8.0 \mathrm{~Hz}, \mathrm{H}_{\mathrm{b}}(\mathrm{II})\right), 2.65\left(1 \mathrm{H}, \mathrm{dd},{ }^{2} J=18.0,{ }^{3} J=5.0 \mathrm{~Hz}, \mathrm{H}_{\mathrm{a}}(\mathrm{II})\right), 2.43(1 \mathrm{H}$, $\left.\mathrm{dd},{ }^{2} J=18.0,{ }^{3} \mathrm{~J}=4.0 \mathrm{~Hz}, \mathrm{H}_{\mathrm{a}}(\mathrm{I})\right) ;{ }^{13} \mathrm{C}$ NMR (100 MHz, DMSO-d 6 ) $\delta 178.0(\mathrm{C}=\mathrm{O}$ imide, I $), 177.8$ ( $\mathrm{C}=\mathrm{O}$ imide, $\mathrm{II}), 176.8(\mathrm{C}=\mathrm{O}$ imide, $\mathrm{II}), 176.6(\mathrm{C}=\mathrm{O}$ imide, $\mathrm{I}), 167.4(\mathrm{C}=\mathrm{O}$ amide, $\mathrm{I}), 162.0$ $(\mathrm{C}(\mathrm{OH})=\mathrm{N}, \mathrm{II}), 116.2(\mathrm{CN}, \mathrm{II}), 115.8(\mathrm{CN}, \mathrm{I}), 58.9\left(\mathrm{CH}_{\mathrm{x}}, \mathrm{II}\right), 58.3\left(\mathrm{CH}_{\mathrm{x}}, \mathrm{I}\right), 34.9\left(\mathrm{CH}_{\mathrm{a}} \mathrm{H}_{\mathrm{b}}, \mathrm{I}\right), 34.2$ $\left(\mathrm{CH}_{\mathrm{a}} \mathrm{H}_{\mathrm{b}}, \mathrm{II}\right), 23.7\left(\mathrm{CH}_{\mathrm{e}} \mathrm{H}_{\mathrm{f}}, \mathrm{I}\right), 23.6\left(\mathrm{CH}_{\mathrm{e}} \mathrm{H}_{\mathrm{f}}, \mathrm{II}\right)$; HRMS m/z calcd. for $\mathrm{C}_{7} \mathrm{H}_{8} \mathrm{~N}_{4} \mathrm{O}_{3} \mathrm{Na}(\mathrm{M}+\mathrm{Na})^{+}$: 219.0491, found : 219.0494; Anal. Calcd. for $\mathrm{C}_{7} \mathrm{H}_{8} \mathrm{~N}_{4} \mathrm{O}_{3}$ : C, 42.86; H, 4.11; N, 28.56; O, 24.47. Found: C, 42.75; H, 4.25; N, 28.65; O, 24.59. 
2-Cyano- $N$ '-(1-methyl-2,5-dioxopyrrolidin-3-yl)acetohydrazide (8b). Yield 56\%; white solid; mp 121-122 ${ }^{\circ} \mathrm{C}$, IR (KBr, $\left.v \mathrm{~cm}^{-1}\right) 3348(\mathrm{w}), 3308(\mathrm{~m}), 3198(\mathrm{w}), 2258(\mathrm{~m}), 1786(\mathrm{w}), 1705$ (s), 1654 (s); ${ }^{1} \mathrm{H}$ NMR (400 MHz, DMSO-d $\left.d_{6}\right) \delta 9.69\left(1 \mathrm{H}, \mathrm{d},{ }^{3} J=5.5 \mathrm{~Hz}, \mathrm{H}_{\mathrm{d}}(\mathrm{I})\right), 9.29(1 \mathrm{H}, \mathrm{br} \mathrm{s}$, $\left.\mathrm{H}_{\mathrm{d}}(\mathrm{II})\right), 5.78\left(2 \mathrm{H}, \mathrm{m}, \mathrm{H}_{\mathrm{c}}(\mathrm{I}, \mathrm{II})\right), 4.08-3.99\left(2 \mathrm{H}, \mathrm{m}, \mathrm{H}_{\mathrm{x}}(\mathrm{I}, \mathrm{II})\right), 3.98\left(1 \mathrm{H}, \mathrm{d},{ }^{2} J=18.0 \mathrm{~Hz}, \mathrm{H}_{\mathrm{f}}(\mathrm{II})\right)$, $3.87\left(1 \mathrm{H}, \mathrm{d},{ }^{2} J=18.0 \mathrm{~Hz}, \mathrm{H}_{\mathrm{e}}(\mathrm{II})\right), 3.63\left(1 \mathrm{H}, \mathrm{d},{ }^{2} J=18.0 \mathrm{~Hz}, \mathrm{H}_{\mathrm{e}}(\mathrm{I})\right), 3.58\left(1 \mathrm{H}, \mathrm{d},{ }^{2} J=18.0 \mathrm{~Hz}\right.$, $\left.\mathrm{H}_{\mathrm{f}}(\mathrm{I})\right), 2.87-2.75\left(8 \mathrm{H}, \mathrm{m}, \mathrm{H}_{\mathrm{b}}(\mathrm{I}), \mathrm{H}_{\mathrm{b}}(\mathrm{II}), \mathrm{CH}_{3}(\mathrm{I}, \mathrm{II})\right), 2.67\left(1 \mathrm{H}, \mathrm{dd},{ }^{2} J=18.0,{ }^{3} J=4.0 \mathrm{~Hz}, \mathrm{H}_{\mathrm{a}}(\mathrm{II})\right)$, $2.54\left(1 \mathrm{H}, \mathrm{dd},{ }^{2} J=18.0{ }^{3} \mathrm{~J}=4.0 \mathrm{~Hz}, \mathrm{H}_{\mathrm{a}}(\mathrm{I})\right) ;{ }^{13} \mathrm{C}$ NMR $\left(100 \mathrm{MHz}, \mathrm{DMSO}-d_{6}\right) \delta 176.5(\mathrm{C}=\mathrm{O}$ imide, II), $176.3(\mathrm{C}=\mathrm{O}$ imide, I), $175.5(\mathrm{C}=\mathrm{O}$ imide, $\mathrm{I}), 175.3(\mathrm{C}=\mathrm{O}$ imide, $\mathrm{II}), 162.0(\mathrm{C}=\mathrm{O}$ amide, $\mathrm{I})$, $161.9(\mathrm{C}(\mathrm{OH})=\mathrm{N}, \mathrm{II}), 116.0(\mathrm{CN}, \mathrm{I}), 115.8(\mathrm{CN}, \mathrm{II}), 57.7\left(\mathrm{CH}_{\mathrm{x}}, \mathrm{I}\right), 57.2\left(\mathrm{CH}_{\mathrm{x}}, \mathrm{II}\right), 33.7\left(\mathrm{CH}_{\mathrm{a}} \mathrm{H}_{\mathrm{b}}\right.$, I), 33.0 $\left(\mathrm{CH}_{\mathrm{a}} \mathrm{H}_{\mathrm{b}}, \mathrm{II}\right), 24.3\left(\mathrm{CH}_{\mathrm{e}} \mathrm{H}_{\mathrm{f}}, \mathrm{II}\right), 24.2\left(\mathrm{CH}_{\mathrm{e}} \mathrm{H}_{\mathrm{f}}, \mathrm{I}\right), 23.7\left(\mathrm{CH}_{3}, \mathrm{II}\right), 23.5\left(\mathrm{CH}_{3}, \mathrm{I}\right) ; \mathrm{HRMS} \mathrm{m} / \mathrm{z}$ calcd. for $\mathrm{C}_{8} \mathrm{H}_{10} \mathrm{~N}_{4} \mathrm{O}_{3} \mathrm{Na}(\mathrm{M}+\mathrm{Na})^{+}: 233.0651$, found : 233.0651; Anal. Calcd. for $\mathrm{C}_{8} \mathrm{H}_{10} \mathrm{~N}_{4} \mathrm{O}_{3}: \mathrm{C}$, 45.71; H, 4.80; N, 26.66; O, 22.84. Found: C, 45.49; H, 4.88; N, 26.41; O, 22.78.

2-Cyano- $N$ '-(1-ethyl-2,5-dioxopyrrolidin-3-yl)acetohydrazide (8c). Yield 44\%; white solid; mp 99-100 ${ }^{\circ} \mathrm{C}$, IR (KBr, $\left.v \mathrm{~cm}^{-1}\right) 3348(\mathrm{w}), 3306$ (m) , 3267(m), 2258 (m), 1774 (s), 1701 (s), $1624(\mathrm{~m}) ;{ }^{1} \mathrm{H}$ NMR $\left(400 \mathrm{MHz}, \mathrm{DMSO}-d_{6}\right) \delta 9.78\left(1 \mathrm{H}\right.$, br s, $\left.\mathrm{H}_{\mathrm{d}}(\mathrm{I})\right), 9.22\left(1 \mathrm{H}\right.$, br s, $\left.\mathrm{H}_{\mathrm{d}}(\mathrm{II})\right), 5.79$ $\left(2 \mathrm{H}, \mathrm{m}, \mathrm{H}_{\mathrm{c}}(\mathrm{I}, \mathrm{II})\right), 4.20\left(1 \mathrm{H}, \mathrm{dd},{ }^{3} J=8.0\right.$ and $\left.4.0 \mathrm{~Hz}, \mathrm{H}_{\mathrm{x}}(\mathrm{II})\right), 4.15\left(1 \mathrm{H}, \mathrm{dd},{ }^{3} J=8.0\right.$ and $4.0 \mathrm{~Hz}$, $\left.\mathrm{H}_{\mathrm{x}}(\mathrm{I})\right), 3.90\left(1 \mathrm{H}, \mathrm{d},{ }^{2} J=18.0 \mathrm{~Hz}, \mathrm{H}_{\mathrm{f}}(\mathrm{II})\right), 3.85\left(1 \mathrm{H}, \mathrm{d},{ }^{2} J=18.0 \mathrm{~Hz}, \mathrm{H}_{\mathrm{e}}(\mathrm{II})\right), 3.65\left(1 \mathrm{H}, \mathrm{d},{ }^{2} J=18.0\right.$ $\left.\mathrm{Hz}, \mathrm{H}_{\mathrm{f}}(\mathrm{I})\right), 3.58\left(1 \mathrm{H}, \mathrm{d},{ }^{2} J=18.0 \mathrm{~Hz}, \mathrm{H}_{\mathrm{e}}(\mathrm{I})\right), 3.40\left(4 \mathrm{H}, \mathrm{q},{ }^{3} J=7.0 \mathrm{~Hz}, \mathrm{CH}_{2}(\mathrm{I}, \mathrm{II})\right), 2.82\left(1 \mathrm{H}, \mathrm{dd},{ }^{2} J\right.$ $\left.=18.0,{ }^{3} J=8.0 \mathrm{~Hz}, \mathrm{H}_{\mathrm{b}}(\mathrm{I})\right), 2.80\left(1 \mathrm{H}, \mathrm{dd},{ }^{2} J=18.0,{ }^{3} J=8.0 \mathrm{~Hz}, \mathrm{H}_{\mathrm{b}}(\mathrm{II})\right), 2.64\left(1 \mathrm{H}, \mathrm{dd},{ }^{2} J=18.0\right.$, $\left.{ }^{3} J=4.0 \mathrm{~Hz}, \mathrm{H}_{\mathrm{a}}(\mathrm{II})\right), 2.40\left(1 \mathrm{H}, \mathrm{dd},{ }^{2} J=18.0,{ }^{3} J=4.0 \mathrm{~Hz}, \mathrm{H}_{\mathrm{a}}(\mathrm{I})\right), 1.02\left(6 \mathrm{H}, \mathrm{t},{ }^{3} J=7.0 \mathrm{~Hz}, \mathrm{CH}_{3}(\mathrm{I}\right.$, II)); ${ }^{13} \mathrm{C}$ NMR (100 MHz, DMSO- $\left.d_{6}\right) \delta 176.2(\mathrm{C}=\mathrm{O}$ imide, II), $176.0(\mathrm{C}=\mathrm{O}$ imide, I), 175.2 $(\mathrm{C}=\mathrm{O}$ imide, $\mathrm{I}), 175.1(\mathrm{C}=\mathrm{O}$ imide, $\mathrm{II}), 167.3(\mathrm{C}=\mathrm{O}$ amide, $\mathrm{I}), 162.0(\mathrm{C}(\mathrm{OH})=\mathrm{N}, \mathrm{II}), 116.4(\mathrm{CN}$, II), $115.8(\mathrm{CN}, \mathrm{I}), 57.7\left(\mathrm{CH}_{\mathrm{x}}, \mathrm{II}\right), 57.1\left(\mathrm{CH}_{\mathrm{x}}, \mathrm{I}\right), 33.8\left(\mathrm{CH}_{2}, \mathrm{I}, \mathrm{II}\right), 33.0\left(\mathrm{CH}_{\mathrm{a}} \mathrm{H}_{\mathrm{b}}, \mathrm{II}\right), 32.8\left(\mathrm{CH}_{\mathrm{a}} \mathrm{H}_{\mathrm{b}}\right.$, I), $23.7\left(\mathrm{CH}_{\mathrm{e}} \mathrm{H}_{\mathrm{f}}, \mathrm{I}\right), 23.5\left(\mathrm{CH}_{\mathrm{e}} \mathrm{H}_{\mathrm{f}}, \mathrm{II}\right), 12.6\left(\mathrm{CH}_{3}, \mathrm{I}, \mathrm{II}\right)$; HRMS $\mathrm{m} / \mathrm{z}$ calcd. for $\mathrm{C}_{9} \mathrm{H}_{12} \mathrm{~N}_{4} \mathrm{O}_{3} \mathrm{Na}$ $(\mathrm{M}+\mathrm{Na})^{+}:$247.0808, found : 247.0807; Anal. Calcd. for $\mathrm{C}_{9} \mathrm{H}_{12} \mathrm{~N}_{4} \mathrm{O}_{3}: \mathrm{C}, 48.21 ; \mathrm{H}, 5.39 ; \mathrm{N}, 24.99$; O, 21.41. Found: C, 48.37; H, 5.43; N, 25.07; O, 21.13.

2-Cyano- $N$ '-(2,5-dioxo-1-phenylpyrrolidin-3-yl)acetohydrazide (8d). Yield 55\%; white solid; $\mathrm{mp}$ 179-180 ${ }^{\circ} \mathrm{C}$, IR (KBr, $\left.v \mathrm{~cm}^{-1}\right) 3483(\mathrm{~m}), 3288(\mathrm{~m}), 3211$ (m), $2258(\mathrm{~m}), 1784$ (s), 1710 (s), $1680(\mathrm{~s}) ;{ }^{1} \mathrm{H}$ NMR $\left(400 \mathrm{MHz}, \mathrm{DMSO}-d_{6}\right) \delta 9.77\left(1 \mathrm{H}, \mathrm{d},{ }^{3} J=5.0 \mathrm{~Hz}, \mathrm{H}_{\mathrm{d}}(\mathrm{I})\right), 9.17(1 \mathrm{H}, \mathrm{br} \mathrm{s}$, $\left.\mathrm{H}_{\mathrm{d}}(\mathrm{II})\right), 7.53-7.25\left(10 \mathrm{H}, \mathrm{m}, \mathrm{H}_{\text {arom. }}(\mathrm{I}, \mathrm{II})\right), 5.88\left(2 \mathrm{H}, \mathrm{m}, \mathrm{H}_{\mathrm{c}}(\mathrm{I}, \mathrm{II})\right), 4.20\left(1 \mathrm{H}, \mathrm{dd},{ }^{3} \mathrm{~J}=8.0\right.$ and 4.0 $\left.\mathrm{Hz}, \mathrm{H}_{\mathrm{x}}(\mathrm{II})\right), 4.17\left(1 \mathrm{H}, \mathrm{dd},{ }^{3} J=8.0\right.$ and $\left.4.0 \mathrm{~Hz}, \mathrm{H}_{\mathrm{x}}(\mathrm{I})\right), 3.90\left(1 \mathrm{H}, \mathrm{d},{ }^{2} J=19.0 \mathrm{~Hz}, \mathrm{H}_{\mathrm{f}}(\mathrm{II})\right), 3.87$ $\left(1 \mathrm{H}, \mathrm{d},{ }^{2} J=19.0 \mathrm{~Hz}, \mathrm{H}_{\mathrm{e}}(\mathrm{II})\right), 3.67\left(1 \mathrm{H}, \mathrm{d},{ }^{2} J=18.0 \mathrm{~Hz}, \mathrm{H}_{\mathrm{f}}(\mathrm{I})\right), 3.62\left(1 \mathrm{H}, \mathrm{d},{ }^{2} J=18.0 \mathrm{~Hz}, \mathrm{H}_{\mathrm{e}}(\mathrm{I})\right)$, $3.05\left(1 \mathrm{H}, \mathrm{dd},{ }^{2} J=18.0,{ }^{3} J=8.0 \mathrm{~Hz}, \mathrm{H}_{\mathrm{b}}(\mathrm{I})\right), 3.00\left(1 \mathrm{H}, \mathrm{dd},{ }^{2} J=18.0,{ }^{3} J=8.0 \mathrm{~Hz}, \mathrm{H}_{\mathrm{b}}(\mathrm{II})\right), 2.85$ $\left(1 \mathrm{H}, \mathrm{dd},{ }^{2} J=18.0,{ }^{3} J=4.0 \mathrm{~Hz}, \mathrm{H}_{\mathrm{a}}(\mathrm{II})\right), 2.69\left(1 \mathrm{H}, \mathrm{dd},{ }^{2} \mathrm{~J}=18.0,{ }^{3} \mathrm{~J}=4.0 \mathrm{~Hz}, \mathrm{H}_{\mathrm{a}}(\mathrm{I})\right) ;{ }^{13} \mathrm{C}$ NMR $\left(100 \mathrm{MHz}, \mathrm{DMSO}-\mathrm{d}_{6}\right) \delta 175.7(\mathrm{C}=\mathrm{O}$ imide, II $), 175.4(\mathrm{C}=\mathrm{O}$ imide, I), $174.6(\mathrm{C}=\mathrm{O}$ imide, $\mathrm{I})$, $174.4(\mathrm{C}=\mathrm{O}$ imide, II), $167.4(\mathrm{C}(\mathrm{OH})=\mathrm{N}, \quad \mathrm{II}), 162.1(\mathrm{C}=\mathrm{O}$ amide, I), 132.2 (Cipso, I), 132.1 (Cipso, II), 128.9 ( $\mathrm{CH}_{\text {arom... }}$ II), 128.8 ( $\mathrm{CH}_{\text {arom. }}$, I), $128.3\left(\mathrm{CH}_{\text {arom., }} \mathrm{II}\right), 128.2\left(\mathrm{CH}_{\text {arom. }}\right.$, I), 127.0 $\left(\mathrm{CH}_{\text {arom., }}\right.$ I), $126.9\left(\mathrm{CH}_{\text {arom. }}\right.$, II $), 116.4(\mathrm{CN}, \mathrm{I}), 115.8(\mathrm{CN}, \mathrm{II}), 57.9\left(\mathrm{CH}_{\mathrm{x}}, \mathrm{II}\right), 57.4\left(\mathrm{CH}_{\mathrm{x}}, \mathrm{I}\right), 34.0$ $\left(\mathrm{CH}_{\mathrm{a}} \mathrm{H}_{\mathrm{b}}, \mathrm{I}\right), 33.4\left(\mathrm{CH}_{\mathrm{a}} \mathrm{H}_{\mathrm{b}}, \mathrm{II}\right), 23.8\left(\mathrm{CH}_{\mathrm{e}} \mathrm{H}_{\mathrm{f}}, \mathrm{I}\right), 23.5\left(\mathrm{CH}_{\mathrm{e}} \mathrm{H}_{\mathrm{f}}, \mathrm{II}\right)$; HRMS $\mathrm{m} / \mathrm{z}$ calcd. for 
$\mathrm{C}_{13} \mathrm{H}_{12} \mathrm{~N}_{4} \mathrm{O}_{3} \mathrm{Na}(\mathrm{M}+\mathrm{Na})^{+}$: 295.0811, found : 295.0807; Anal. Calcd. for $\mathrm{C}_{13} \mathrm{H}_{12} \mathrm{~N}_{4} \mathrm{O}_{3}$ : C, 57.35; H, 4.44; N, 20.58; O, 17.63. Found: C, 57.25; H, 4.46; N, 20.29; O, 17.87.

\section{General procedure for preparation of compounds 9}

To a solution of compounds 8a-d $(10 \mathrm{mmol})$ in ethanol $(20 \mathrm{~mL})$ was added phenyl isothiocyanate $(10 \mathrm{mmol})$. The resulting mixture was brought to reflux for 12 hours. The solid obtained after cooling was filtered and crystallized from ethanol to give compounds $9 \mathbf{9 a - d}$.

$\boldsymbol{N}$-[5-(2-Amino-2-oxoethyl)-4-oxo-3-phenyl-2-thioxoimidazolidin-1-yl]-2-cyanoacetamide

(9a). Yield 57\%; white solid; mp 158-160 ${ }^{\circ} \mathrm{C}$; IR (KBr, $v \mathrm{~cm}^{-1}$ ) 3340 (m), 3284 (m), 2262 (m), 1710 (m), 1697 (s), 1670 (s), 1512 (m); ${ }^{1} \mathrm{H}$ NMR (400 MHz, DMSO-d $\left.)_{6}\right) 11.25\left(1 \mathrm{H}, \mathrm{s}, \mathrm{H}_{\mathrm{d}}\right), 7.56$ $\left(2 \mathrm{H}, \mathrm{s}, \mathrm{NH}_{2}\right), 7.60-7.35\left(\mathrm{~m}, 5 \mathrm{H}, \mathrm{H}_{\text {arom. }}\right), 4.67\left(1 \mathrm{H}, \mathrm{t},{ }^{3} \mathrm{~J}=4.0 \mathrm{~Hz}, \mathrm{H}_{\mathrm{x}}\right), 3.89\left(1 \mathrm{H}, \mathrm{d},{ }^{2} \mathrm{~J}=18.0 \mathrm{~Hz}\right.$, $\left.\mathrm{H}_{\mathrm{e}}\right), 3.85\left(1 \mathrm{H}, \mathrm{d},{ }^{2} J=18.0 \mathrm{~Hz}, \mathrm{H}_{\mathrm{f}}\right), 2.87\left(2 \mathrm{H}, \mathrm{d},{ }^{3} \mathrm{~J}=4.0 \mathrm{~Hz}, \mathrm{H}_{\mathrm{a}}, \mathrm{H}_{\mathrm{b}}\right) ;{ }^{13} \mathrm{C} \mathrm{NMR}(100 \mathrm{MHz}$, DMSO- $\left.d_{6}\right) \delta 183.2(\mathrm{C}=\mathrm{S}), 171.5\left(\mathrm{NH}_{2} \mathrm{C}=\mathrm{O}\right), 169.7(\mathrm{C}=\mathrm{O}$ thioimidazole $), 162.2(\mathrm{NNHC}=\mathrm{O})$, 133.8 (Cipso), $128.9\left(\mathrm{CH}_{\text {arom. }}\right), 128.8\left(\mathrm{CH}_{\text {arom. }}\right), 128.5\left(\mathrm{CH}_{\text {arom. }}\right), 115.1(\mathrm{CN}), 59.5\left(\mathrm{CH}_{\mathrm{x}}\right), 34.9$ $\left(\mathrm{CH}_{\mathrm{a}} \mathrm{H}_{\mathrm{b}}\right), 23.9\left(\mathrm{CH}_{\mathrm{e}} \mathrm{H}_{\mathrm{f}}\right)$; HRMS $\mathrm{m} / \mathrm{z}$ calcd. for $\mathrm{C}_{14} \mathrm{H}_{13} \mathrm{~N}_{5} \mathrm{O}_{3} \mathrm{~S}(\mathrm{M}+\mathrm{H})^{+}$: 332.0824, found : 332.0817; Anal. Calcd. for $\mathrm{C}_{14} \mathrm{H}_{13} \mathrm{~N}_{5} \mathrm{O}_{3} \mathrm{~S}$ : C, 50.75; H, 3.95; N, 21.14; O, 14.49; S, 9.68. Found: C, 50.84; H, 4.09; N, 21.35; O, 14.98; S, 9.74.

2-Cyano- $\mathrm{N}$-\{5-[2-(methylamino)-2-oxoethyl]-4-oxo-3-phenyl-2-thioxoimidazolidin-1-

yl \}acetamide (9b). Yield 66\%; white solid; mp 222-224 ${ }^{\circ} \mathrm{C}$, IR (KBr, v cm $\left.{ }^{-1}\right) 3379$ (m), 3286 (w), 2260(m), 1766 (s), 1654 (s), $1500(\mathrm{~m}) ;{ }^{1} \mathrm{H}$ NMR (400 MHz, DMSO-d $\left.d_{6}\right) 11.20\left(1 \mathrm{H}, \mathrm{s}, \mathrm{H}_{\mathrm{d}}\right)$, $8.05\left(1 \mathrm{H}, \mathrm{q},{ }^{3} \mathrm{~J}=4.5 \mathrm{~Hz}, \mathrm{NH}\right), 7.65-7.35\left(5 \mathrm{H}, \mathrm{m}, \mathrm{H}_{\text {arom. }}\right), 4.75\left(1 \mathrm{H}, \mathrm{t},{ }^{3} \mathrm{~J}=4.0 \mathrm{~Hz}, \mathrm{H}_{\mathrm{x}}\right), 3.95(1 \mathrm{H}$, $\left.\mathrm{d},{ }^{2} J=18.0 \mathrm{~Hz}, \mathrm{H}_{\mathrm{e}}\right), 3.88\left(1 \mathrm{H}, \mathrm{d},{ }^{2} J=18.0 \mathrm{~Hz}, \mathrm{H}_{\mathrm{f}}\right), 2.93\left(2 \mathrm{H}, \mathrm{d},{ }^{3} J=4.0 \mathrm{~Hz}, \mathrm{H}_{\mathrm{a}}, \mathrm{H}_{\mathrm{b}}\right), 2.65(3 \mathrm{H}, \mathrm{d}$, $\left.J=4.5 \mathrm{~Hz}, \mathrm{NCH}_{3}\right) ;{ }^{13} \mathrm{C} \mathrm{NMR}\left(100 \mathrm{MHz}, \mathrm{DMSO}-d_{6}\right) \delta 183.1(\mathrm{C}=\mathrm{S}), 171.3\left(\mathrm{NH}\left(\mathrm{CH}_{3}\right) \mathrm{C}=\mathrm{O}\right)$, $167.8\left(\mathrm{C}=\mathrm{O}\right.$ thioimidazole), $162.1(\mathrm{NNHC}=\mathrm{O}), 133.7$ (Cipso), $128.8\left(\mathrm{CH}_{\text {arom. }}\right), 128.7\left(\mathrm{CH}_{\text {arom. }}\right)$, 128.4 ( $\left.\mathrm{CH}_{\text {arom. }}\right), 115.0(\mathrm{CN}), 59.5\left(\mathrm{CH}_{\mathrm{x}}\right), 33.3\left(\mathrm{CH}_{\mathrm{a}} \mathrm{H}_{\mathrm{b}}\right), 25.5\left(\mathrm{NCH}_{3}\right), 23.8\left(\mathrm{CH}_{\mathrm{e}} \mathrm{H}_{\mathrm{f}}\right) ; \mathrm{HRMS} \mathrm{m} / \mathrm{z}$ calcd. for $\mathrm{C}_{15} \mathrm{H}_{16} \mathrm{~N}_{5} \mathrm{O}_{3} \mathrm{~S}(\mathrm{M}+\mathrm{H})^{+}:$346.0971, found : 346.0974; Anal. Calcd. for $\mathrm{C}_{15} \mathrm{H}_{15} \mathrm{~N}_{5} \mathrm{O}_{3} \mathrm{~S}$ : C, 52.16; H, 4.38; N, 20.28; O, 13.90; S, 9.28. Found: C, 51.95; H, 4.29; N, 20.37; O, 14.24; S, 9.15 .

\section{2-Cyano- $N$-\{5-[2-(ethylamino)-2-oxoethyl]-4-oxo-3-phenyl-2-thioxoimidazolidin-1-}

yl \}acetamide (9c). Yield 53\%; white solid; mp 145-146 ${ }^{\circ} \mathrm{C}, \mathrm{IR}\left(\mathrm{KBr}, v \mathrm{~cm}^{-1}\right) 3369$ (m), 3169 (w), 2258 (m), 1766 (s), 1708 (s), 1647 (s), 1500 (m); ${ }^{1} \mathrm{H}$ NMR(400MHz, DMSO-d $\left.{ }_{6}\right) \delta 11.20$ $\left(1 \mathrm{H}, \mathrm{s}, \mathrm{H}_{\mathrm{d}}\right), 8.05\left(1 \mathrm{H}, \mathrm{t},{ }^{3} J=4.5 \mathrm{~Hz}, \mathrm{NHC}_{2} \mathrm{H}_{5}\right), 7.60-7.30\left(5 \mathrm{H}, \mathrm{m}, \mathrm{H}_{\text {arom. }}\right), 4.75\left(1 \mathrm{H}, \mathrm{t},{ }^{3} J=4.0\right.$ $\left.\mathrm{Hz}, \mathrm{H}_{\mathrm{x}}\right), 3.89\left(1 \mathrm{H}, \mathrm{d},{ }^{2} J=18.0 \mathrm{~Hz}, \mathrm{H}_{\mathrm{e}}\right), 3.85\left(1 \mathrm{H}, \mathrm{d},{ }^{2} J=18.0 \mathrm{~Hz}, \mathrm{H}_{\mathrm{f}}\right), 3.10\left(2 \mathrm{H}, \mathrm{m}, \mathrm{NCH}_{2}\right), 2.86$ $\left(2 \mathrm{H}, \mathrm{d},{ }^{3} \mathrm{~J}=4.0 \mathrm{~Hz}, \mathrm{H}_{\mathrm{a}}, \mathrm{H}_{\mathrm{b}}\right), 1.00\left(3 \mathrm{H}, \mathrm{t},{ }^{3} \mathrm{~J}=7.0 \mathrm{~Hz}, \mathrm{CH}_{3}\right) ;{ }^{13} \mathrm{C}$ NMR $\left(100 \mathrm{MHz}, \mathrm{DMSO}-d_{6}\right) \delta$ $183.1(\mathrm{C}=\mathrm{S}), 171.4\left(\mathrm{NH}\left(\mathrm{C}_{2} \mathrm{H}_{5}\right) \mathrm{C}=\mathrm{O}\right), 167.1(\mathrm{C}=\mathrm{O}$ thioimidazole $), 162.2(\mathrm{NNHC}=\mathrm{O}), 133.8$ (Cipso), $128.9\left(\mathrm{CH}_{\text {arom. }}\right), 128.8\left(\mathrm{CH}_{\text {arom. }}\right), 128.5\left(\mathrm{CH}_{\text {arom. }}\right), 115.1(\mathrm{CN}), 59.6\left(\mathrm{CH}_{\mathrm{x}}\right), 33.5\left(\mathrm{CH}_{\mathrm{a}} \mathrm{H}_{\mathrm{b}}\right)$, $33.1\left(\mathrm{NCH}_{2}\right), 23.9\left(\mathrm{CH}_{\mathrm{e}} \mathrm{H}_{\mathrm{f}}\right), 14.5\left(\mathrm{CH}_{3}\right)$; HRMS m/z calcd. for $\mathrm{C}_{16} \mathrm{H}_{18} \mathrm{~N}_{5} \mathrm{O}_{3} \mathrm{~S}(\mathrm{M}+\mathrm{H})^{+}: 360.3541$, found : 360.3543; Anal. Calcd. for $\mathrm{C}_{20} \mathrm{H}_{17} \mathrm{~N}_{5} \mathrm{O}_{3} \mathrm{~S}$ : C, 53.47; H, 4.77; N, 19.49; O, 13.35; S, 8.92. Found: C, 52.70; H, 4.73; N, 19.53; O, 13.82; S, 8.73. 


\section{$\mathrm{N}$-[5-[2-Anilino-2-oxoethyl)-4-oxo-3-phenyl-2-thioxoimidazolidin-1-yl]-2-cyanoacetamide)}

(9d). Yield 57\%; white solid; mp 258-259 ${ }^{\circ} \mathrm{C}$; IR (KBr, $\left.v \mathrm{~cm}^{-1}\right) 3335$ (m), 3190 (m), 2260 (m), 1763 (s), 1710 (s), 1668 (s), 1502 (m); ${ }^{1} \mathrm{H}$ NMR (400 MHz, DMSO-d $)_{6} \delta 11.29\left(1 \mathrm{H}, \mathrm{s}, \mathrm{H}_{\mathrm{d}}\right.$ ), $10.20(1 \mathrm{H}, \mathrm{s}, \mathrm{NHPh})$ ), 7.60-7.45 (4H, m, $\left.\mathrm{H}_{\text {arom. }}\right), 7.50\left(1 \mathrm{H}, \mathrm{tt},{ }^{3} J=7.0 \mathrm{~Hz},{ }^{4} \mathrm{~J}=1.5 \mathrm{~Hz}, \mathrm{H}_{\text {arom. }}\right)$, 7.40-7.30 (4H, m, $\left.\mathrm{H}_{\text {arom. }}\right), 7.05\left(1 \mathrm{H}, \mathrm{tt},{ }^{3} \mathrm{~J}=7.5 \mathrm{~Hz},{ }^{4} \mathrm{~J}=1.0 \mathrm{~Hz}, \mathrm{H}_{\text {arom. }}\right), 4.81\left(1 \mathrm{H}, \mathrm{dd},{ }^{3} J=4.0\right.$ $\mathrm{Hz}$ and $\left.3.5 \mathrm{~Hz}, \mathrm{H}_{\mathrm{x}}\right), 3.90\left(1 \mathrm{H}, \mathrm{d},{ }^{2} J=18.0 \mathrm{~Hz}, \mathrm{H}_{\mathrm{e}}\right), 3.85\left(1 \mathrm{H}, \mathrm{d},{ }^{2} J=18.0 \mathrm{~Hz}, \mathrm{H}_{\mathrm{f}}\right), 3.17(1 \mathrm{H}$, dd, $\left.{ }^{2} J=18.0 \mathrm{~Hz},{ }^{3} J=3.5 \mathrm{~Hz}, \mathrm{H}_{\mathrm{a}}\right), 3.13\left(1 \mathrm{H}, \mathrm{dd},{ }^{2} J=18.0 \mathrm{~Hz},{ }^{3} J=4.0 \mathrm{~Hz}, \mathrm{H}_{\mathrm{b}}\right) ;{ }^{13} \mathrm{C}$ NMR $(100$ MHz, DMSO- $\left.d_{6}\right) \delta 183.1(\mathrm{C}=\mathrm{S}), 171.3(\mathrm{NH}(\mathrm{Ph}) \mathrm{C}=\mathrm{O}), 166.6(\mathrm{C}=\mathrm{O}$ thioimidazole $), 162.3$ $(\mathrm{NNHC}=\mathrm{O}), 138.7$ (Cipso), 133.7 (Cipso), $129.0\left(\mathrm{CH}_{\text {arom. }}\right), 128.8\left(\mathrm{CH}_{\text {arom. }}\right), 128.7\left(\mathrm{CH}_{\text {arom. }}\right)$, 128.5 ( $\left.\mathrm{CH}_{\text {arom. }}\right), 123.4$ ( $\left.\mathrm{CH}_{\text {arom. }}\right), 119.1\left(\mathrm{CH}_{\text {arom. }}\right), 115.1(\mathrm{CN}), 59.5\left(\mathrm{CH}_{\mathrm{x}}\right), 34.5\left(\mathrm{CH}_{\mathrm{a}} \mathrm{H}_{\mathrm{b}}\right), 23.9$ $\left(\mathrm{CH}_{\mathrm{e}} \mathrm{H}_{\mathrm{f}}\right)$; HRMS m/z calcd. for $\mathrm{C}_{20} \mathrm{H}_{18} \mathrm{~N}_{5} \mathrm{O}_{3} \mathrm{~S}(\mathrm{M}+\mathrm{H})^{+}$: 408.1131, found : 408.1130; Anal. Calcd. for $\mathrm{C}_{20} \mathrm{H}_{17} \mathrm{~N}_{5} \mathrm{O}_{3} \mathrm{~S}$ : C, 58.96; H, 4.21; N, 17.19; O, 11.78; S, 7.87. Found: C, 58.83; H, 4.26; N, $17.14 ; \mathrm{O}, 12.03 ; \mathrm{S}, 7.36$

2-Cyano- $N$ '(2,5-dioxo-1-phenylpyrrolidin-3-yl)-3-phenylacrylo hydrazide (10). To a solution of $\mathbf{8 d}(1 \mathrm{mmol})$ in ethanol $(20 \mathrm{~mL})$ containing a catalytic amount of piperidine, benzaldehyde (10 mmol) was added. The reaction mixture was heated at reflux for 12 hours. The resulting solid formed was collected by filtration. Yield 58\%; yellow solid; mp $163-165{ }^{\circ} \mathrm{C}$; IR $\left(\mathrm{KBr}, v \mathrm{~cm}^{-1}\right)$ 3298 (w), 3059 (w), 1780 (s), 1708 (s), 1651 (m), 1604 (m); ${ }^{1} \mathrm{H}$ NMR (400 MHz, DMSO-d 6 ) $\delta 10.25\left(1 \mathrm{H}\right.$, br s, $\left.\mathrm{H}_{\mathrm{d}}\right), 8.16\left(1 \mathrm{H}, \mathrm{s}, \mathrm{H}_{\mathrm{g}}\right), 7.95\left(2 \mathrm{H}, \mathrm{m}, \mathrm{H}_{\text {arom. }}\right), 7.65-7.55\left(3 \mathrm{H}, \mathrm{m}, \mathrm{H}_{\text {arom. }}\right), 7.50(2 \mathrm{H}$, $\left.\mathrm{t},{ }^{3} J=7.5 \mathrm{~Hz}, \mathrm{H}_{\text {arom. }}\right), 7.30\left(2 \mathrm{H}, \mathrm{d},{ }^{3} J=7.5 \mathrm{~Hz}, \mathrm{H}_{\text {arom. }}\right), 6.09\left(1 \mathrm{H}, \mathrm{br} \mathrm{s}, \mathrm{H}_{\mathrm{c}}\right) ; 4.28\left(1 \mathrm{H}, \mathrm{dd},{ }^{3} J=8.5\right.$ and $\left.4.0 \mathrm{~Hz}, \mathrm{H}_{\mathrm{x}}\right), 3.10\left(1 \mathrm{H}, \mathrm{dd},{ }^{2} J=18.0,{ }^{3} J=8.5 \mathrm{~Hz}, \mathrm{H}_{\mathrm{a}}\right), 2.80\left(1 \mathrm{H}, \mathrm{dd},{ }^{2} J=18.0,{ }^{3} J=4.0 \mathrm{~Hz}\right.$, $\left.\mathrm{H}_{\mathrm{b}}\right) ;{ }^{13} \mathrm{C}$ NMR (100 MHz, DMSO-d 6$) \delta 175.3(\mathrm{C}=\mathrm{O}$ imide), $174.7(\mathrm{C}=\mathrm{O}$ imide), 161.0 $(\mathrm{NNHC}=\mathrm{O}), 150.8(\mathrm{CH}=\mathrm{C}), 132.4$ (Cipso), 132.2 (Cipso), $131.8\left(\mathrm{CH}_{\text {arom. }}\right), 130.0\left(\mathrm{CH}_{\text {arom. }}\right)$, $129.2\left(\mathrm{CH}_{\text {arom. }}\right), 128.9$ ( $\left.\mathrm{CH}_{\text {arom. }}\right), 128.5\left(\mathrm{CH}_{\text {arom. }}\right), 126.9\left(\mathrm{CH}_{\text {arom. }}\right), 115.8(\mathrm{CN}), 104.8(\mathrm{C}=\mathrm{CH})$, $57.3\left(\mathrm{CH}_{\mathrm{x}}\right), 34.4\left(\mathrm{CH}_{\mathrm{a}} \mathrm{H}_{\mathrm{b}}\right)$; HRMS $\mathrm{m} / \mathrm{z}$ calcd. for $\mathrm{C}_{20} \mathrm{H}_{16} \mathrm{~N}_{4} \mathrm{O}_{3}: 383.1106$, found : 383.1120 .

$\mathrm{N}$-(4-(2-Anilino-2-oxoethyl)-5-oxo-1-phenyl-2-thioxopyrrolidin-3-yl)-2-cyano-3-phenyl-

acrylamide (11). A solution of compound 10 (1 mmol.) in ethanol $(20 \mathrm{~mL})$ was refluxed with a large excess of phenyl isothiocyanate during 5 hours. The yellow solid formed is collected by filtration. Yield 45\%; yellow solid; mp 216-218 ${ }^{\circ} \mathrm{C}$; IR ( $\left.\mathrm{KBr}, \mathrm{v} \mathrm{cm}^{-1}\right) 3258(\mathrm{w}), 3129$ (w), 2220 (m), 1753 (s), 1686 (s), 1647 (m), 1595 (m), 1271 (w); ${ }^{1} \mathrm{H}$ NMR (400 MHz, DMSO-d 6 ) $\delta 11.62$ $\left(1 \mathrm{H}, \mathrm{s}, \mathrm{H}_{\mathrm{d}}\right), 10.19(1 \mathrm{H}, \mathrm{s}, \mathrm{NHPh}), 8.32\left(1 \mathrm{H}, \mathrm{s}, \mathrm{H}_{\mathrm{g}}\right), 7.95\left(2 \mathrm{H}, \mathrm{d},{ }^{3} J=6.5 \mathrm{~Hz}, \mathrm{H}_{\text {arom. }}\right), 7.65-7.55$ $\left(7 \mathrm{H}, \mathrm{m}, \mathrm{H}_{\text {arom. }}\right), 7.50\left(1 \mathrm{H}, \mathrm{t},{ }^{3} J=7.0 \mathrm{~Hz}, \mathrm{H}_{\text {arom. }}\right), 7.45\left(2 \mathrm{H}, \mathrm{d},{ }^{3} J=7.5 \mathrm{~Hz}, \mathrm{H}_{\text {arom. }}\right), 7.35\left(2 \mathrm{H}, \mathrm{t},{ }^{3} J\right.$ $\left.=7.5 \mathrm{~Hz}, \mathrm{H}_{\text {arom. }}\right), 7.05\left(1 \mathrm{H}, \mathrm{t},{ }^{3} J=7.5 \mathrm{~Hz}, \mathrm{H}_{\text {arom. }}\right), 4.87\left(1 \mathrm{H}, \mathrm{t},{ }^{3} J=4.0 \mathrm{~Hz}, \mathrm{H}_{\mathrm{x}}\right), 3.20\left(2 \mathrm{H}, \mathrm{dd},{ }^{2} J=\right.$ $\left.17.9 \mathrm{~Hz},{ }^{3} \mathrm{~J}=4.0 \mathrm{~Hz}, \mathrm{H}_{\mathrm{a}}\right) ; 3.17\left(1 \mathrm{H}, \mathrm{dd},{ }^{2} \mathrm{~J}=17.9,{ }^{3} \mathrm{~J}=4.0 \mathrm{~Hz}, \mathrm{H}_{\mathrm{b}}\right) ;{ }^{13} \mathrm{C} \mathrm{NMR}(100 \mathrm{MHz}$, DMSO- $\left.d_{6}\right) \delta 184.7(\mathrm{C}=\mathrm{S}), 170.0(\mathrm{C}=\mathrm{O}$ thioimidazole $), 166.0(\mathrm{C}=\mathrm{O}$ amide $), 160.7(\mathrm{NNHC}=\mathrm{O})$, 153.1 ( $\mathrm{CH}=\mathrm{C}$ ), 138.8 (Cipso), 133.7 (Cipso), 131.3 (Cipso), $130.2\left(\mathrm{CH}_{\text {arom. }}\right), 129.3\left(\mathrm{CH}_{\text {arom. }}\right.$ ), 128.9 ( $\left.\mathrm{CH}_{\text {arom. }}\right), 128.8$ ( $\left.\mathrm{CH}_{\text {arom. }}\right), 128.7$ ( $\left.\mathrm{CH}_{\text {arom. }}\right), 128.6$ ( $\left.\mathrm{CH}_{\text {arom. }}\right), 128.4$ ( $\left.\mathrm{CH}_{\text {arom. }}\right), 123.2$ $\left(\mathrm{CH}_{\text {arom. }}\right), 119.0\left(\mathrm{CH}_{\text {arom. }}\right), 115.3(\mathrm{CN}), 103.3(\mathrm{C}=\mathrm{CH}), 59.9\left(\mathrm{CH}_{\mathrm{x}}\right), 35.0\left(\mathrm{CH}_{\mathrm{a}} \mathrm{H}_{\mathrm{b}}\right) ; \mathrm{HRMS} \mathrm{m} / \mathrm{z}$ calcd. for $\mathrm{C}_{27} \mathrm{H}_{22} \mathrm{~N}_{5} \mathrm{O}_{3} \mathrm{~S}(\mathrm{M}+\mathrm{H})^{+}:$496.1443, found : 496.1448 . 


\section{General procedure for preparation of compounds 13}

To a mixture of 2-mercapto benzimidazoles 12 (1 mmol), appropriate substituted maleimide 5 $(1.1 \mathrm{mmol})$ and sulphuric acid $(1.1 \mathrm{mmol})$ was added glacial acetic acid $(5 \mathrm{~mL})$. The resulting solution was heated under reflux for 10 hours. After cooling, the solution was treated with $\mathrm{NH}_{4} \mathrm{OH}$ until neutralization. The resulting solid formed was collected by filtration, washed several times with water, dried and crystallized from ethanol to give the corresponding compounds 13a-d.

2-(3-Oxo-2,3-dihydro[1,3]thiazolo[3,2-a]benzimidazol-2-yl) acetamide (13a). Yield 43\%; white solid; mp 190-192 ${ }^{\circ} \mathrm{C}$, IR ( $\mathrm{KBr}, v \mathrm{~cm}^{-1}$ ) 3227 (w), 1780 (s), 1708 (s); ${ }^{1} \mathrm{H}$ NMR (400MHz, DMSO-d 6 ) $\delta 12.70(1 \mathrm{H}, \mathrm{s}, \mathrm{NH}), 11.50(1 \mathrm{H}, \mathrm{s}, \mathrm{NH}), 7.42\left(2 \mathrm{H}, \mathrm{m}, \mathrm{H}_{\text {arom. }}\right), 7.25$ (2H, m, $\left.\mathrm{H}_{\text {arom. }}\right)$, $4.73\left(1 \mathrm{H}, \mathrm{dd},{ }^{3} \mathrm{~J}=9.5\right.$ and $\left.5.5 \mathrm{~Hz}, \mathrm{H}_{\mathrm{x}}\right), 3.23\left(1 \mathrm{H}, \mathrm{dd},{ }^{2} J=18.0 \mathrm{~Hz},{ }^{3} \mathrm{~J}=9.5 \mathrm{~Hz}, \mathrm{H}_{\mathrm{a}}\right), 2.95(1 \mathrm{H}$, dd, $\left.{ }^{2} J=18,{ }^{3} J=5.5 \mathrm{~Hz}, \mathrm{H}_{\mathrm{b}}\right) ;{ }^{13} \mathrm{C} \mathrm{NMR}\left(100 \mathrm{MHz}, \mathrm{DMSO}-d_{6}\right) \delta 176.4(\mathrm{C}=\mathrm{O}$ amide $), 176.3(\mathrm{C}=\mathrm{O}$ thioimidazole $), 147.5(\mathrm{C}=\mathrm{N}), 143.4\left(\mathrm{C}_{\mathrm{ipso}}\right), 135.1\left(\mathrm{C}_{\mathrm{ipso}}\right), 121.9\left(\mathrm{CH}_{\text {arom. }}\right), 121.2\left(\mathrm{CH}_{\text {arom. }}\right), 117.5$ $\left(\mathrm{CH}_{\text {arom. }}\right), 110.5\left(\mathrm{CH}_{\text {arom. }}\right), 42.6\left(\mathrm{CH}_{\mathrm{x}}\right), 37.5\left(\mathrm{CH}_{\mathrm{a}} \mathrm{H}_{\mathrm{b}}\right)$; HRMS $\mathrm{m} / \mathrm{z}$ calcd. for $\mathrm{C}_{11} \mathrm{H}_{10} \mathrm{~N}_{3} \mathrm{O}_{2} \mathrm{~S}$ $(\mathrm{M}+\mathrm{H})^{+}:$248.0504, found : 248.0494; Anal. Calcd. for $\mathrm{C}_{11} \mathrm{H}_{9} \mathrm{~N}_{3} \mathrm{O}_{2} \mathrm{~S}: \mathrm{C}, 53.43 ; \mathrm{H}, 3.67$; N, 16.99; O, 12.94; S, 12.97. Found: C, 53.04; H, 3.75; N, 16.80; O, 13.08; S, 12.88.

$\mathrm{N}$-Methyl-2-(3-oxo-2,3-dihydro[1,3]thiazolo[3,2-a]benzimidazol-2-yl) acetamide (13b). Yield 46\%; white solid; mp 156-157 ${ }^{\circ} \mathrm{C}$, IR (KBr, $\left.v \mathrm{~cm}^{-1}\right) 3195$ (m), 1766 (s), 1697 (s); ${ }^{1} \mathrm{H}$ NMR $\left(400 \mathrm{MHz}, \mathrm{DMSO}-d_{6}\right) \delta 12.61\left(1 \mathrm{H}\right.$, br s, NH), $7.40\left(2 \mathrm{H}, \mathrm{m}, \mathrm{H}_{\text {arom. }}\right), 7.30\left(2 \mathrm{H}, \mathrm{m}, \mathrm{H}_{\text {arom. }}\right), 4.76$ $\left(1 \mathrm{H}, \mathrm{dd},{ }^{3} J=9.5\right.$ and $\left.5.0 \mathrm{~Hz}, \mathrm{H}_{\mathrm{x}}\right), 3.31\left(1 \mathrm{H}, \mathrm{dd},{ }^{2} J=18.0 \mathrm{~Hz},{ }^{3} J=9.5 \mathrm{~Hz}, \mathrm{H}_{\mathrm{a}}\right), 2.90\left(1 \mathrm{H}, \mathrm{dd},{ }^{2} J=\right.$ $\left.18.0 \mathrm{~Hz},{ }^{3} \mathrm{~J}=5.0 \mathrm{~Hz}, \mathrm{H}_{\mathrm{b}}\right), 2.85\left(3 \mathrm{H}, \mathrm{s}, \mathrm{CH}_{3}\right) ;{ }^{13} \mathrm{C} \mathrm{NMR}\left(100 \mathrm{MHz}, \mathrm{DMSO}-d_{6}\right) \delta 175.4(\mathrm{C}=\mathrm{O}$ amide), $175.2(\mathrm{C}=\mathrm{O}$ thioimidazole $), 147.6(\mathrm{C}=\mathrm{N}), 134.5\left(\mathrm{C}_{\mathrm{ipso}}\right), 132.1\left(\mathrm{C}_{\mathrm{ipso}}\right), 122.2\left(\mathrm{CH}_{\text {arom. }}\right)$, $109.4\left(\mathrm{CH}_{\text {arom. }}\right), 41.2\left(\mathrm{CH}_{\mathrm{x}}\right), 36.5\left(\mathrm{CH}_{\mathrm{a}} \mathrm{H}_{\mathrm{b}}\right), 24.9\left(\mathrm{CH}_{3}\right)$; HRMS m/z calcd. for $\mathrm{C}_{12} \mathrm{H}_{12} \mathrm{~N}_{3} \mathrm{O}_{2} \mathrm{~S}$ $(\mathrm{M}+\mathrm{H})^{+}:$262.2805, found : 262.2803; Anal. Calcd. for $\mathrm{C}_{12} \mathrm{H}_{11} \mathrm{~N}_{3} \mathrm{O}_{2} \mathrm{~S}: \mathrm{C}, 55.16 ; \mathrm{H}, 4.24 ; \mathrm{N}$, 16.08; O, 12.25; S, 12.27. Found: C, 54.59; H, 4.23; N, 15.92; O, 12.64; S, 12.60.

2-(3-Oxo-2,3-dihydro[1,3]thiazolo[3,2-a]benzimidazol-2-yl)- $N$-phenylacetamide (13d). Yield 42\%; white solid; mp 258-260 ${ }^{\circ} \mathrm{C}$, IR $\left(\mathrm{KBr}, v \mathrm{~cm}^{-1}\right) 3169$ (m), 1751 (s), 1668 (s); ${ }^{1} \mathrm{H}$ NMR $\left(400 \mathrm{MHz}, \mathrm{DMSO}-d_{6}\right) \delta 12.63\left(1 \mathrm{H}_{2} . \mathrm{s}, \mathrm{NH}\right), 7.56-7.13\left(9 \mathrm{H}, \mathrm{m}, \mathrm{H}_{\text {arom. }}\right), 4.86\left(1 \mathrm{H}, \mathrm{dd},{ }^{3} J=9.5\right.$ and $\left.5.0 \mathrm{~Hz}, \mathrm{H}_{\mathrm{x}}\right), 3.46\left(1 \mathrm{H}, \mathrm{dd},{ }^{2} J=18.0 \mathrm{~Hz},{ }^{3} \mathrm{~J}=9.5 \mathrm{~Hz}, \mathrm{H}_{\mathrm{a}}\right), 3.09\left(1 \mathrm{H}, \mathrm{dd},{ }^{2} J=18.0 \mathrm{~Hz},{ }^{3} J=5.0\right.$ $\mathrm{Hz}) ;{ }^{13} \mathrm{C}$ NMR (100 MHz, DMSO- $\left.d_{6}\right) \delta 174.5(\mathrm{C}=\mathrm{O}$ amide), 174.2 (C=O thioimidazole), 148.9 $(\mathrm{C}=\mathrm{N}), 138.9\left(\mathrm{C}_{\mathrm{ipso}}\right), 138.5\left(\mathrm{C}_{\text {ipso }}\right), 130.8\left(\mathrm{C}_{\mathrm{ipso}}\right), 128.7\left(\mathrm{CH}_{\text {arom. }}\right), 124.4\left(\mathrm{CH}_{\text {arom. }}\right), 123.2$ ( $\left.\mathrm{CH}_{\text {arom. }}\right)$, $121.6\left(\mathrm{CH}_{\text {arom. }}\right), 115.4\left(\mathrm{CH}_{\text {arom. }}\right), 41.4\left(\mathrm{CH}_{\mathrm{x}}\right), 36.6\left(\mathrm{CH}_{\mathrm{a}} \mathrm{H}_{\mathrm{b}}\right)$; HRMS $\mathrm{m} / \mathrm{z}$ calcd. for $\mathrm{C}_{17} \mathrm{H}_{14} \mathrm{~N}_{3} \mathrm{O}_{2} \mathrm{~S}(\mathrm{M}+\mathrm{H})^{+}:$324.0813, found : 324.0807; Anal. Calcd. for $\mathrm{C}_{17} \mathrm{H}_{13} \mathrm{~N}_{3} \mathrm{O}_{2} \mathrm{~S}: \mathrm{C}$, 48.45; $\mathrm{H}$, 3.59; N, 9.97; O, 22.78; S, 15.22. Found: C, 48.05; H, 3.61; N, 9.94; O, 22.82; S, 15.53.

\section{References}

1. He, S.; Kuang, R.; Venkatamaran, R.; Tu, J.; Truong, T. M.; Chan, H. T.; Groutas, W. Bioorg. Med. Chem. 2000, 8, 1713. 
2. Fuentes, J.; Salameh, B. A. B.; Pradera, A.; Fernandez de Cordoba, F. J.; Gash, G. Tetrahedron 2006, 62, 97 and references cited therein.

3. (a) Roue, N.; Bergman, J Tetrahedron 1999, 55, 14729. (b) Cherouvrier, J. R.; Carreaux, F.; Bazureau, J. P. Tetrahedron Lett. 2002, 43, 8745. Renault, S.; Bertrand, S.; Carreaux, F.; Bazureau, J. P. J. Comb. Chem. 2007, 9, 935. (c) Porwal, S.; Chauhan, S. S.; Chauhan, P. M. S.; Shakya, N.; Verma, A.; Gupta, S. J. Med. Chem. 2009, 52, 5793.

4. Muccioli, G. G.; Fazio, N.; Sciba, G. K. E.; Poppitz, W.; Cannata, F.; Poupaert, J. H.; Wouters, J.; Lambert, D. M. J. Med. Chem. 2006, 49, 417.

5. Sawyers, C.; Jung, M. E.; Chen, C. D.; Ouk, S.; Welsbie, D.; Tran, C.; Wongvipat, J.; Yoo, D. WO 2006124118.

6. Claussner, A.; Goubet, F.; Teutsch, J. G. WO 1997 19064. Thurieau, C.; Poitout, L. WO 2001 09090. Binet, J.; Boubia, B.; Chaput, E.; Edgar, A.; Ou, K.; Ratel, P.; Samreth, S.; Thomas, D. WO 2004 031160. Wan, Y.; Pan, S.; Zhang, G.; Wang, X.; Xie, Y. F.; Jiang, J.; Phillips, D.; Yang, Y. WO 2009123948.

7. Lewalter, J.; Rottmaier, L.; Merten, R.; Dunwald, W.; Schulte, B. EP 19800013727.

8. Bélai, I. Tetrahedron Lett. 2003, 44, 7475. Severinsen, R.; Lau, J. F.; Bondensgaard, K.; Hansen, B. S.; Begtrup, M.; Ankersen, M. Bioorg. Med. Chem. Lett. 2004, 14, 317.

9. Maiti, B.; Chanda, K.; Sun, C. M. Org. Lett. 2009, 11, 4826.

10. Lin, M. -L.; Sun, C. -M. Tetrahedron Lett. 2003, 44, 8739.

11. White, A. W.; Curtin, N. I.; Eastman, B. W.; Golding, B. T.; Hostomsky, Z.; Kyle, S.; Li, J.; Maegley, K. A.; Skalitzky, D. J.; Webber, S. E.; Yu, X. H.; Griffin, R. J. Bioorg. Med. Chem. Lett. 2004, 14, 2433.

12. Ates-Alagoz, Z.; Yildiz, S.; Buyukbingol, E. Chemotherapy 2007, 53, 110.

13. Hong, S. Y.; Kwal, K. W.; Ryu, C. K.; Kang, S. J.; Chung, K. H. Bioorg. Med. Chem. Lett. 2008, 16, 644.

14. Grimaudo, S.; Raimondi, M. V.; Capone, F.; Chimirri, A.; Poretto, F.; Monforte, A. M.; Simoni, D.; Tolomeo, M. Eur. J. Cancer 2001, 37, 122.

15. Hamdy, N. A.; Abdel-Aziz, H. A.; Farag, A. M.; Fakhr, I. M. I. Monatsh. Chem. 2007, 138, 1001.

16. Bouzroura, S.; Hammal, L.; Nedjar-Kolli, B.; Balegroune, F.; Hamadène, M.; Poulain, S. Synth. Commun. 2007, 38, 448.

17. Bondock, S.; Tarhoni, A. E-G.; Fadda, A. A. Arkivoc 2006, (ix), 113.

18. Dyachenko, V.; Dyachenko, V. D.; Rusanov, B. E. Russ. J. Org. Chem. 2007, 43, 83. Mohareb, R. M.; Aziz, S. A.; Abdel-Sayed, N. I.; El-Banna, A. H. J. Chem. Research (M) 1999, 101.

19. Alper, A. E.; Taurins, A. Can. J. Chem. 1967, 45, 2903-2912. Hayashibe, S.; Kamikubo, T.; Tsukamoto, S. -I.; Sakamoto, S. Heterocycles 2004, 62, 815.

20. Khaliullin, F. A.; Klen, E. E. Chem. Heterocycl. Comp. 2009, 45, 876 and references cited therein.

21. Fadda, A. A.; Bondock, S.; Rabie, R.; Etman, H. A. Turk. J. Chem. 2008, 32, 259. 